\title{
Usefulness of brain FDG PET/CT imaging in pediatric patients with suspected autoimmune encephalitis from a prospective study
}

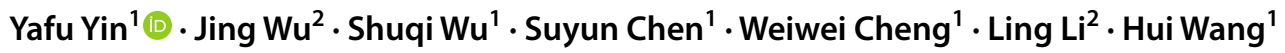

Received: 15 September 2021 / Accepted: 3 December 2021 / Published online: 23 December 2021

(c) The Author(s) 2021

\begin{abstract}
Purpose Early diagnosis and treatment are of paramount importance for pediatric patients with autoimmune encephalitis (AE). The aim is to evaluate the usefulness of FDG PET/CT in pediatric patients with suspected AE from a prospective study. Methods The prospective study was conducted over a period of 23.5 months from May 14, 2019, to April 30, 2021. All patients ( $<18$-year-old) were hospitalized at the department of pediatric neurology and met the criteria of clinical suspected AE. The children underwent the tests of blood samplings, CSF, EEG, MRI, and ${ }^{18}$ F-FDG PET/CT. The criteria for FDG $\mathrm{PET} / \mathrm{CT}$ diagnosis of AE were large lobar hypometabolism with or without focal hypermetabolism found on PET/CT. The clinical final diagnosis of AE includes seropositive and seronegative AE based on the diagnostic criteria.

Results One hundred four pediatric inpatients ( 57 boys, 47 girls) were included, of which 58 children were diagnosed with $\mathrm{AE}$ (seropositive, 16; seronegative, 42), 45 children were diagnosed with non-AE, and one boy remained indeterminate diagnosis. Large lobar hypometabolism was found in 61 children, of which 54 (88.5\%) children were finally diagnosed with AE. The sensitivity, specificity, and accuracy of FDG PET/CT for diagnosis of AE were $93.1 \%, 84.4 \%$, and $89.3 \%$, respectively, with a positive predictive value of $88.5 \%$ and a negative predictive value of $90.5 \%$. The most common involved with hypometabolism was the parietal lobe, followed by occipital and frontal lobes, finally the temporal lobe on PET/CT in children with AE. Conclusion Brain FDG PET/CT imaging has high specificity, sensitivity, and accuracy for diagnosis of AE in clinical suspected $\mathrm{AE}$ children.

Trial registration.

Clinical Trials.gov. NCT02969213. Registered 17 October 2016.
\end{abstract}

Keywords Autoimmune encephalitis $\cdot$ FDG PET/CT $\cdot$ Lobar hypometabolism $\cdot$ Suspected AE

Yafu Yin and Jing Wu contributed equally to this work.

This article is part of the Topical Collection on Neurology

Yafu Yin

yinyafu@xinhuamed.com.cn; yinyf-2001@163.com

$\triangle$ Ling Li

liling@xinhuamed.com.cn

$\triangle$ Hui Wang

wanghui@xinhuamed.com.cn

1 Department of Nuclear Medicine, Yangpu District, Xinhua Hospital Affiliated To Shanghai Jiao Tong University School of Medicine, Kongjiang Road 1665, Shanghai City 200092, China

2 Department of Pediatric Neurology, Yangpu District, Xinhua Hospital Affiliated To Shanghai Jiao Tong University School of Medicine, Kongjiang Road 1665, Shanghai City 200092, China

\section{Introduction}

Autoimmune encephalitis (AE) generally refers to a group of severe inflammatory brain disease due to abnormal autoimmunity mechanism. The archetypical syndrome of $\mathrm{AE}$ includes variable combinations of neuropsychiatric and behavioral changes, psychosis, seizures, memory and cognitive deficits, abnormal movements, dysautonomia, and a decreased level of consciousness [1]. AE is one of the most challenging clinical conditions to diagnose because of the heterogeneity in disease manifestations and diagnostic test results, overlap in clinical presentations between $\mathrm{AE}$, other inflammatory brain diseases, metabolic diseases, and psychiatric disorders. It is especially difficult for children because of the complexity of normal behavioral changes during childhood and the limited capacity of younger children to describe their symptoms. Early diagnosis is paramount for 
pediatric patients with $\mathrm{AE}$ because early initiation of immunotherapy is associated with a better prognosis [2-8], even severely affected children can have profound improvement when getting early and aggressive treatment $[9,10]$, otherwise worsened prognosis and even permanent neurocognitive deficits happened [11].

In the initial stage, the diagnostic criteria for $\mathrm{AE}$ were dependent on autoantibody testing and response to immunotherapy, while, depending on autoantibody testing, the diagnosis may significantly delay treatment initiation. Moreover, autoantibody testing is not readily available at most institutions, and there might be some autoantibodies have not yet been identified by now. In 2016, an international group developed diagnostic criteria for early diagnosis of $\mathrm{AE}$ in adults, which included the diagnoses of possible, probable, and definite AE to reduce the delay in initiation of therapy due to waiting for autoantibody testing results [12]. The items of criteria described in the report were composed of clinical symptoms, cerebrospinal fluid (CSF) sampling, serum sampling, electroencephalogram (EEG), and MRI. It is a real pity that ${ }^{18} \mathrm{~F}$-FDG PET/CT was not recommended in the criteria as a diagnostic tool for possible $\mathrm{AE}$ or seronegative probable AE. In fact, a significant percentage of $\mathrm{AE}$, mostly more than half, brain MRI shows normal or only non-specific changes [13]. ${ }^{18} \mathrm{~F}-\mathrm{FDG}$ PET/CT shows a much greater sensitivity for detecting an underlying abnormality than MRI in patients with AE [14-16]. Abnormal brain metabolism detected on FDG-PET/CT is most often in early stage of AE, compared to initial EEG, MRI, and CSF studies in inpatients with AE [17].

So far, many works have described the characteristic findings supporting the diagnostic value of ${ }^{18} \mathrm{~F}$-FDG PET/CT in $\mathrm{AE}$ [18-24]. PET imaging was not included in the diagnostic criteria mostly because there was short of robust evidence to accelerate the comprehension and validation of ${ }^{18} \mathrm{~F}$-FDG PET in the emerging field of AE [25], and especially due to the lack of prospective studies. The data is even more limited in pediatric $\mathrm{AE}$, as noted in recently published clinical approach to the diagnosis of $\mathrm{AE}$ in pediatric patient [26].

In this study, a prospective study was conducted and aimed to evaluate the usefulness of FDG PET/CT in pediatric patients with clinical suspected $\mathrm{AE}$.

\section{Methods}

\section{Study design and participants}

This prospective study was conducted over a period of 23.5 months from May 14, 2019, to April 30, 2021, in Xinhua Hospital affiliated to Shanghai Jiao Tong University School of Medicine. The pediatric inpatients $(<18$ yearold), with the following criteria, were considered clinical suspected AE and included: (1) clinical symptoms: seizures, neuropsychiatric changes (e.g. memory and cognitive deficits, behavioral changes, psychosis), movement disorders and dysautonomia, and (2) at least one of the following abnormalities: CSF pleocytosis; CSF-specific oligoclonal bands or elevated CSF IgG index; elevated erythrocyte sedimentation rate, complement protein, antinuclear antibody, immunoglobulin, lymphocyte count, and inflammatory factors in serum sampling; MRI features suggestive of encephalitis; abnormal EEG; positive etiologic test; paraneoplastic antibody.

All the enrolled patients underwent brain ${ }^{18} \mathrm{~F}$-FDG PET/ CT scanning and neuronal autoantibody test in the serum and CSF samplings. Based on the further findings, the pediatric neurologist gave the children further immunotherapy as appropriate [2]. Exclusion criteria were as follows: (1) refuse to perform PET/CT scanning or fail to finish PET/CT scanning, (2) refuse further examination or immunotherapy under no definite diagnosis, and (3) infant with age less than one-year-old.

The criteria of clinical final diagnosis of $\mathrm{AE}$, for the enrolled suspected $\mathrm{AE}$ patients, include the following:

\section{Seropositive AE}

The autoimmune neuronal antibodies were identified in the serum and/or CSF samplings.

\section{Seronegative AE}

The diagnosis was made when all three of the following criteria have been met: (1) none of autoimmune neuronal antibody was identified in the serum or CSF sampling, (2) $\geq 1$ feature present: CSF-specific oligoclonal bands; intrathecal synthesis of immunoglobulin or elevated CSF IgG index; CSF pleocytosis ( $>5$ cells) or elevated protein; brain MRI abnormalities suggestive of AE; effective immunotherapy, and (3) reasonable exclusion of alternative causes.

\section{Brain ${ }^{18}$ F-FDG PET/CT imaging}

Brain ${ }^{18} \mathrm{~F}-\mathrm{FDG}$ PET/CT imaging was performed according to the routine institutional procedures. In brief, all patients fasted from food and sugary drinks for at least $4 \mathrm{~h}$ prior to intravenous injection of approximately $25-185 \mathrm{MBq}{ }^{18} \mathrm{~F}$ FDG based on $3.7 \mathrm{MBq} / \mathrm{kg}$ body weight. All patients rested in a quiet and dim room before and after injection. About 50 min following injection, a brain PET/CT scanning was performed for $10 \mathrm{~min}$ on a Biograph mCT-64 scanner (Siemens, Erlangen, Germany). 


\section{Imaging analysis}

All PET/CT images were independently assessed and reviewed by two nuclear medicine physicians with extensive experience with brain FDG PET/CT imaging.

The criteria for FDG PET/CT diagnosis of AE and nonAE: (1) large lobar hypometabolism with or without focal hypermetabolism found on PET/CT was defined as AE; (2) focal hypometabolism which is consistent with a clinical epileptic seizure, even if combined with extensive area of hypometabolism around, was defined as an epileptogenic focus, or hypometabolism could be explained by tumor or other disease or not were all classified as non-AE; (3) no obvious abnormalities found on PET/CT were classified as non-AE. Large lobar hypometabolism means extensive decreased glucose metabolism in bilateral lobes or more than one unilateral lobe.

\section{Statistical analysis}

Data analysis was performed using the IBM SPSS Statistics 25 software (USA: IBM Corp). Descriptive statistics included the frequency (percentage) for categorical variables and median for continuous variables. Chi-square, fisher's exact, and two-tailed $t$ student test were used to compare the clinical, laboratory, and imaging features between the groups of pediatric patients as appropriate. All tests were two-tailed and $p<0.05$ was considered statistically significant.

\section{Results}

\section{Patient demographics}

In total, 104 pediatric patients ( 57 boys, 47 girls, median age 7 years, range 1-17 years) were recruited in this prospective study. The clinical characteristics of the included children are summarized in Table 1, except for one boy whose diagnosis was indeterminate.

\section{Final definite diagnosis}

Finally, 58 children were diagnosed with AE, of which 16 children were seropositive and 42 were seronegative AE. Forty-five children were diagnosed with non-AE, and one child remained indeterminate diagnosis, who was an 11-year-old boy with neuropsychiatric changes, infection, negative results of CSF, EEG, and MRI, and no response to immunotherapy.

Sixteen seropositive AE children presented with positive neuronal autoantibody test for anti-NMDAR (7 children),
anti-Ri ( 2 children), anti-MOG ( 2 children), anti-Hu and anti-Amphiphysin (1 girl), anti-DPPX (1 girl), anti-GQ1b (1 boy), anti-AMPAR1 (1 boy), and anti-Recoverin (1 girl).

Among 45 non-AE patients, 23 children (51\%) were diagnosed with epilepsy, 9 children were diagnosed with mental retardation, 4 were tic disorder, 3 were headache, and 6 were diagnosed with brain tumor, Niemann-Pick disease, postencephalitis, somatization disorder, syringomyelus, and hydrocephalus, respectively.

\section{Clinical manifestation}

The most common manifestations of the recruited children with definite diagnosis were seizures (61 children, 59.2\%) and neuropsychiatric changes (62 children, $60.2 \%$ ). Neuropsychiatric changes were more often presented in AE children $(70.7 \%)$ than non-AE $(46.7 \%)(p<0.05)$. Twenty-six children presented with both seizures and neuropsychiatric changes, of which 6 children were seropositive $\mathrm{AE}, 13$ were seronegative AE, and 7 were non-AE. Seizures happened with neuropsychiatric changes more often in $\mathrm{AE}$ children $(19 / 32)$ than non-AE $(7 / 29)(p<0.05)$. Less common manifestations were dysautonomia (13 children, $12.6 \%)$ and movement disorders (4 children, 3.9\%). Dysautonomia was relatively more often presented in AE children in this study $(p<0.05)$ (Table 1).

\section{Clinical examination}

Infection, cellular immunity, and inflammatory factors were very common in $\mathrm{AE}$ children with a high positive rate of $94.8 \%, 98.2 \%$, and $85.5 \%$ (Table 1), respectively, which were slightly higher than that in non-AE children $(88.9 \%, 97.5 \%$, and $75.0 \%$ ), but no significant difference reached.

The CSF abnormalities, including CSF-specific oligoclonal bands positive, intrathecal synthesis of immunoglobulin or elevated CSF IgG index, CSF pleocytosis ( $>5$ cells) or elevated protein, were more often positive in $\mathrm{AE}$ than non-AE children $(58.6 \% \& 12.5 \%, p<0.05)$.

The autoantibodies associated with connective tissue disorders and erythrocyte sedimentation rate were less often positive in $\mathrm{AE}$ and non-AE children $(34.5 \%, 21.1 \%$ \& 9.8\%, $14.6 \%$ ); the difference of the former between groups reached statistical significance $(p<0.05)$.

In non-AE children, MR imaging showed non-specific abnormalities in 8 cases, brainstem encephalitis, meningocephalitis, tuberous sclerosis, and hydrocephalus in one case each. In AE children, MR showed non-specific abnormalities in 7 cases, pituitory Rathke cyst in 2 cases, encephalitis and focal cortical dysplasia in one case each.

EEG abnormalities were common in both $\mathrm{AE}$ and non-AE children (65.5\% \& 64.4\%); no significant difference reached $(p<0.05)$. 
Table 1 The clinical characteristics of 103 children with definite diagnosis

\begin{tabular}{|c|c|c|c|c|c|c|}
\hline \multirow[t]{2}{*}{ Characteristic } & \multirow[t]{2}{*}{ Total } & \multicolumn{2}{|l|}{$\mathrm{AE}$} & \multirow[t]{2}{*}{ Non-AE } & \multirow[t]{2}{*}{$p^{* *}$} & \multirow[t]{2}{*}{$p^{* * *}$} \\
\hline & & Seropositive & Seronegative & & & \\
\hline Number & 103 & $\begin{array}{l}58 \\
16(27.6 \%)\end{array}$ & $42(72.4 \%)$ & 45 & - & - \\
\hline Median age (range), years & $7(1-17)$ & $\begin{array}{l}7(1-17) \\
6.5(2-17)\end{array}$ & $7(1-13)$ & $7(1-15)$ & 0.409 & 0.927 \\
\hline Gender & & & & & & \\
\hline Female & $47(45.6 \%)$ & $\begin{array}{l}30(51.7 \%) \\
10(62.5 \%)\end{array}$ & $20(47.6 \%)$ & $17(37.8 \%)$ & 0.311 & 0.169 \\
\hline Male & $56(54.4 \%)$ & $\begin{array}{l}28(48.3 \%) \\
6(37.5 \%)\end{array}$ & $22(52.4 \%)$ & $28(62.2 \%)$ & & \\
\hline Clinical syndromes & & & & & & \\
\hline Seizures & $61(59.2 \%)$ & $\begin{array}{l}32(55.2 \%) \\
7(43.8 \%)\end{array}$ & $25(59.5 \%)$ & $29(64.4 \%)$ & 0.280 & 0.342 \\
\hline Neuropsychiatric changes & $62(60.2 \%)$ & $\begin{array}{l}41(70.7 \%) \\
15(93.8 \%)\end{array}$ & $26(61.9 \%)$ & $21(46.7 \%)$ & 0.017 & 0.013 \\
\hline Movement disorders & $4(3.9 \%)$ & $\begin{array}{l}3(5.2 \%) \\
1(6.3 \%)\end{array}$ & $2(4.8 \%)$ & $1(2.2 \%)$ & 1.000 & 0.630 \\
\hline Dysautonomia & $13(12.6 \%)$ & $\begin{array}{l}11(19.0 \%) \\
3(18.8 \%)\end{array}$ & $8(19.0 \%)$ & $2(4.4 \%)$ & 1.000 & 0.036 \\
\hline Infection & $95(92.2 \%)$ & $\begin{array}{l}55(94.8 \%) \\
15(93.8 \%)\end{array}$ & $40(95.2 \%)$ & $40(88.9 \%)$ & 0.819 & 0.264 \\
\hline $\mathrm{CSF}^{*}$ & $39(40.6 \%)$ & $\begin{array}{l}34(58.6 \%) \\
7(43.8 \%)\end{array}$ & $27(64.3 \%)$ & $5(12.5 \%)$ (7 untested) & 0.156 & 0.000 \\
\hline Erythrocyte sedimentation rate & $18(18.4 \%)$ & $\begin{array}{l}12(21.1 \%) \\
5(31.3 \%)\end{array}$ & $7(17.1 \%)(1$ untested $)$ & $6(14.6 \%)$ (4 untested) & 0.238 & 0.418 \\
\hline Autoantibody ${ }^{\#}$ & $24(24.2 \%)$ & $\begin{array}{l}20(34.5 \%) \\
7(43.8 \%)\end{array}$ & $13(31.0 \%)$ & $4(9.8 \%)$ (4 untested) & 0.359 & 0.005 \\
\hline Cellular immunity & $95(97.9 \%)$ & $\begin{array}{l}56(98.2 \%) \\
16(100 \%)\end{array}$ & $40(97.6 \%)$ (1 untested) & $39(97.5 \%)$ (5 untested) & 0.529 & 0.799 \\
\hline Inflammatory factors & $77(81.1 \%)$ & $\begin{array}{l}47(85.5 \%) \\
16(100 \%)\end{array}$ & $31(79.5 \%)$ (3 untested) & $30(75.0 \%)(5$ untested $)$ & 0.05 & 0.199 \\
\hline EEG & $67(65.0 \%)$ & $\begin{array}{l}38(65.5 \%) \\
9(56.3 \%)\end{array}$ & $29(69.0 \%)$ & $29(64.4 \%)$ & 0.359 & 0.910 \\
\hline MRI & $23(22.3 \%)$ & $\begin{array}{l}11(19.0 \%) \\
2(12.5 \%)\end{array}$ & $9(21.4 \%)$ & $12(26.7 \%)$ & 0.438 & 0.352 \\
\hline
\end{tabular}

${ }^{*}$ CSF abnormalities includes CSF-specific oligoclonal bands; intrathecal synthesis of immunoglobulin or elevated CSF IgG index; CSF pleocytosis ( $>5$ cells) or elevated protein

** This $p$ value is the chi-square test for seropositive and seronegative AE groups

*** This $p$ value is the chi-square test for AE and non-AE groups

\# The term autoantibody here refers to the antibodies that are associated with connective tissue disorders

\section{Application of newly published diagnostic criteria for pediatric AE}

The newly published diagnostic criteria for pediatric AE were developed by the Autoimmune Encephalitis International Working Group in 2020 [26]. The proposed classification criteria for possible pediatric $\mathrm{AE}$, probable antibody-negative pediatric $\mathrm{AE}$, and definite antibody-positive pediatric AE were provided in Table 2 [26]. Among seropositive AE patients, $10(62.5 \%)$ children fulfilled criteria for definite antibody-positive AE; the other 6 children did not meet the criteria mostly due to onset of neurologic and/ or psychiatric symptoms over $>3$ months. Among seronegative AE patients, 13 (31.0\%) children fulfilled criteria for possible AE, 4 (9.5\%) fulfilled criteria for probable antibody-negative $\mathrm{AE}$, and 25 (59.5\%) children did not meet 
Table 2 The proposed classification criteria by AE International Working Group [26]

\begin{tabular}{llll}
\hline Categorical features of AE & Possible AE & Probable antibody-negative AE & Definite antibody-positive AE \\
\hline $\begin{array}{l}\text { Acute or subacute onset: Onset of neurologic and/ } \\
\text { or psychiatric symptoms over } \leq 3 \text { mo in a previously } \\
\text { healthy child }\end{array}$ & Yes & Yes & Yes \\
$\begin{array}{l}\text { Clinical evidence of neurologic dysfunction: Altered } \\
\text { mental status/level of consciousness or EEG with } \\
\text { slowing or epileptiform activity (focal or generalized) }\end{array}$ & $\geq 2$ features present & $\geq 2$ features present & $\geq 2$ features present \\
$\begin{array}{l}\text { Focal neurologic deficits Cognitive difficulties Acute } \\
\text { developmental regression Movement disorder (except } \\
\text { tics) Psychiatric symptoms Seizures not explained by a } \\
\text { previously known seizure disorder or other condition }\end{array}$ & & & \\
$\begin{array}{l}\text { Paraclinical evidence of neuroinflammation: CSF } \\
\text { inflammatory changes (leukocytosis }>5 \text { cells/mm and/ } \\
\text { or oligoclonal banding) MRI features of encephalitis }\end{array}$ & Not available & $\geq 1$ features present & $\geq 1$ features present \\
$\begin{array}{l}\text { Brain biopsy showing inflammatory infiltrates and } \\
\text { excluding other disorders }\end{array}$ & & & \\
$\begin{array}{l}\text { AE serology: Presence in serum and/or CSF of well- } \\
\text { characterized autoantibodies associated with AE }\end{array}$ & Not available & No & \\
$\begin{array}{l}\text { Exclusion of other etiologies: Reasonable exclusion } \\
\text { of alternative causes, including other causes of CNS } \\
\text { inflammation }\end{array}$ & Yes & Yes & \\
\hline
\end{tabular}

the criteria. The proportion of pediatric patients meeting the latest diagnostic criteria was very low, mainly because of the long onset of symptoms, normal or non-specific CSF and MRI findings, and the absence of a brain biopsy.

\section{Brain FDG PET/CT imaging}

All the patients underwent brain FDG PET/CT scanning, of which 9 children underwent scanning twice. The results of 103 primary brain PET/CT imaging were listed in Table 3, except for the boy with indeterminate diagnosis.

Among 103 children with definite diagnosis, large lobar hypometabolism with or without focal hypermetabolism (Figs. 1 and 2) was found on PET/CT in 61 children, of which 54 (88.5\%) children were eventually diagnosed with AE. Normal brain imaging (Fig. 3) was found in 23 children, of which 2 (8.7\%) children were diagnosed with AE. Focal foci with hypometabolism (Fig. 4) were found in 19 children, of which $2(10.5 \%)$ children were diagnosed with AE finally. Among the children with focal foci, epileptogenic foci were found on PET/CT in 10 children. The sensitivity, specificity, and accuracy of FDG PET/CT for diagnosis of AE were $93.1 \%$ (54/58), 84.4\% (38/45), and $89.3 \%$ $(92 / 103)$, respectively, with a positive predictive value of $88.5 \%(54 / 61)$ and a negative predictive value of $90.5 \%$ (38/42). The boy with indeterminate diagnosis showed lobar hypometabolism with focal hypermetabolism.
Table 3 The results of brain $\mathrm{PET} / \mathrm{CT}$ imaging

\begin{tabular}{|c|c|c|c|c|c|c|}
\hline \multirow[t]{2}{*}{$\mathrm{PET} / \mathrm{CT}$} & & \multicolumn{2}{|l|}{$\mathrm{AE}(58)$} & \multirow[t]{2}{*}{ Non-AE (45) } & \multirow[t]{2}{*}{$p^{* *}$} & \multirow[t]{2}{*}{$p^{* * *}$} \\
\hline & & Seropositive(16) & Seronegative(42) & & & \\
\hline \multirow[t]{4}{*}{$\mathrm{AE}(61)$} & Lobar A* (23) & $21(36.2 \%)$ & & $2(4.4 \%)$ & 0.031 & 0.000 \\
\hline & & $2(12.5 \%)$ & $19(45.2 \%)$ & & & \\
\hline & Lobar B* ${ }^{*}(38)$ & $33(56.9 \%)$ & & $5(11.1 \%)$ & 0.036 & 0.000 \\
\hline & & $13(81.3 \%)$ & $20(47.6 \%)$ & & & \\
\hline \multirow[t]{4}{*}{ Non-AE (42) } & Focal foci (19) & $2(3.4 \%)$ & & $17(37.8 \%)$ & 0.479 & 0.000 \\
\hline & & $1(6.3 \%)$ & $1(2.4 \%)$ & & & \\
\hline & Normal (23) & $2(3.4 \%)$ & & $21(46.7 \%)$ & 1.000 & 0.000 \\
\hline & & 0 & $2(4.8 \%)$ & & & \\
\hline
\end{tabular}

*Lobar A stands for lobar hypometabolism without focal hypermetabolism; Lobar B stands for lobar hypometabolism with focal hypermetabolism

** This $p$ value is the chi-square test for seropositive and seronegative AE groups

**** This $p$ value is the chi-square test for $\mathrm{AE}$ and non-AE groups 


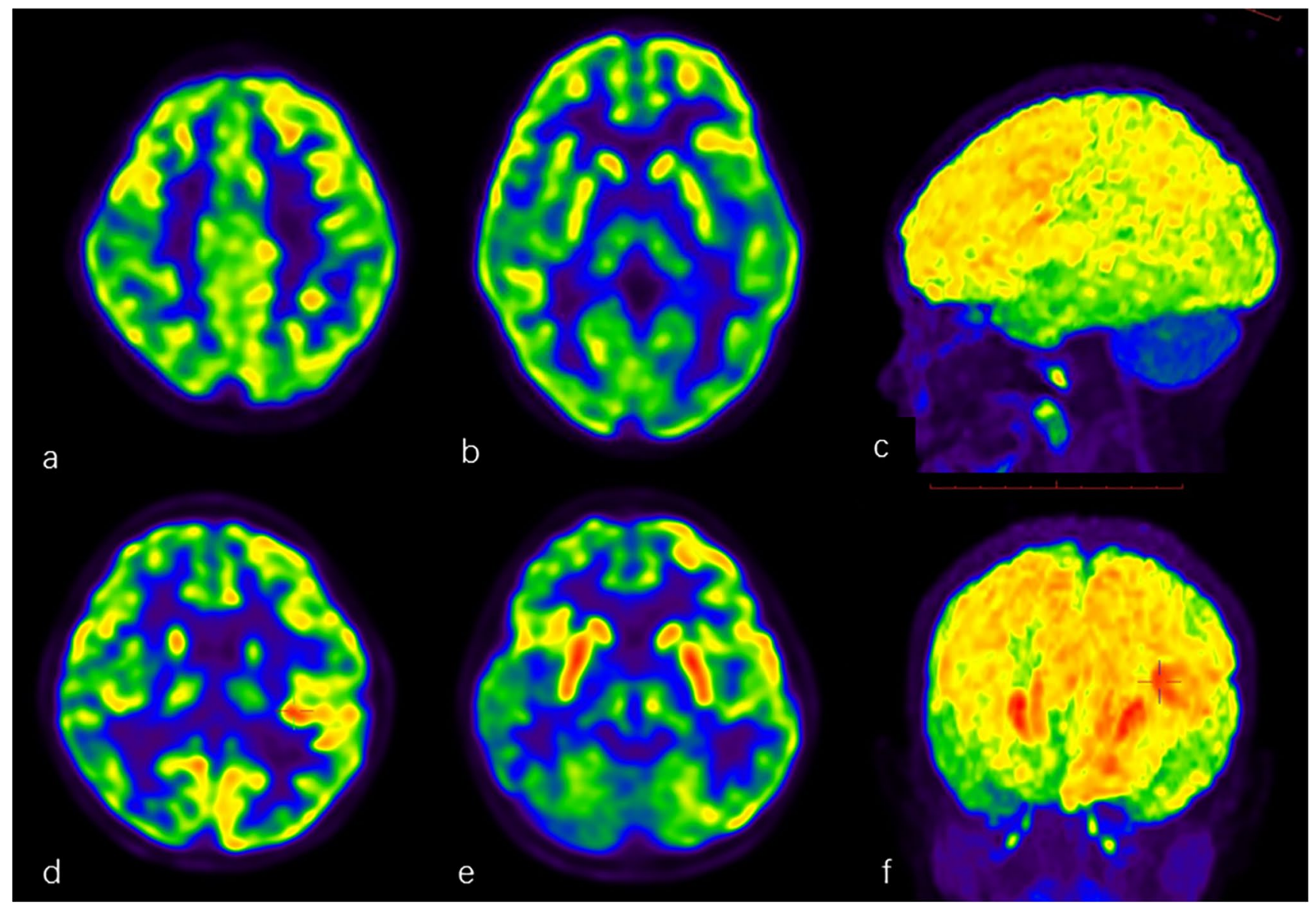

Fig. 1 Top row: A 4-year-old girl presenting with fewer words and personality changes. Anti-MOG antibody test was positive. Brain ${ }^{18} \mathrm{~F}-$ FDG PET/CT showed large-scale lobar hypometabolism in bilateral parietal and occipital lobes without focal hypermetabolism $(\mathbf{a}, \mathbf{b}, \mathbf{c})$. Bottom row: An 8-year-old girl presented with seizures, personality, and cognitive changes for more than 3 months, who was finally diag-

In total, lobar hypometabolism with focal hypermetabolism was found on PET/CT in 38 children with definite diagnosis, of which 33 (86.8\%) children were diagnosed with AE composed of 8 focal cortical hypermetabolism and 27 hypermetabolism in basal ganglia. Among children involved with lobar hypometabolism without focal hypermetabolism, 91.3\% (21/23) were diagnosed with AE. In the 58 AE children, the most common involved with hypometabolism was the parietal lobe $(55,94.8 \%)$, followed by the occipital (35, $60.3 \%)$ and frontal lobes $(34,58.6 \%)$, finally the temporal lobe $(22,37.9 \%)$ on PET/CT.

In seronegative $\mathrm{AE}$ children, abnormal metabolism involved 3 children in one lobe (bilateral) $(7.1 \%), 16$ children in two lobes (38.1\%), 12 children in three lobes $(28.6 \%), 8$ children in four lobes $(19.0 \%), 15$ children in basal ganglia (35.7\%). In seropositive AE children, abnormal metabolism involved 1 child in one lobe (bilateral) nosed with anti-NMDAR AE. Brain ${ }^{18}$ F-FDG PET/CT showed largescale hypometabolism in bilateral parietal, occipital, temporal, and right frontal lobes $(\mathbf{d}, \mathbf{e}, \mathbf{f})$ with focal hypermetabolism in left frontal and fronto-temporal lobe (the cross: $\mathbf{d}, \mathbf{f}$ ) and bilateral basal ganglia $(\mathbf{e}, \mathbf{f})$. c, f MIP map of FDG PET

(6.3\%), 5 children in two lobes $(31.3 \%), 4$ children in three lobes (25.0\%), 5 children in four lobes (31.3\%), and 12 children in basal ganglia $(75.0 \%)$.

Four children with AE showed normal or focal hypometabolism on PET/CT, three of which mainly presented with developmental retardation, especially in language, and had a longer course than one year. One child presented with seizure; PET/CT showed focal hypometabolism in right central sulcus mainly in the parietal lobe (Fig. 4).

Six children had the repeated brain PET/CT scanning 1 to 7 months after immunotherapy, hypometabolism got well recovered (Fig. 2). Three patients had the scanning shortly (19 days to 2.5 months) after therapy, hypermetabolism of basal ganglia appeared or got more obvious and more extensive hypometabolism presented. All these 9 patients were diagnosed with seronegative AE. 


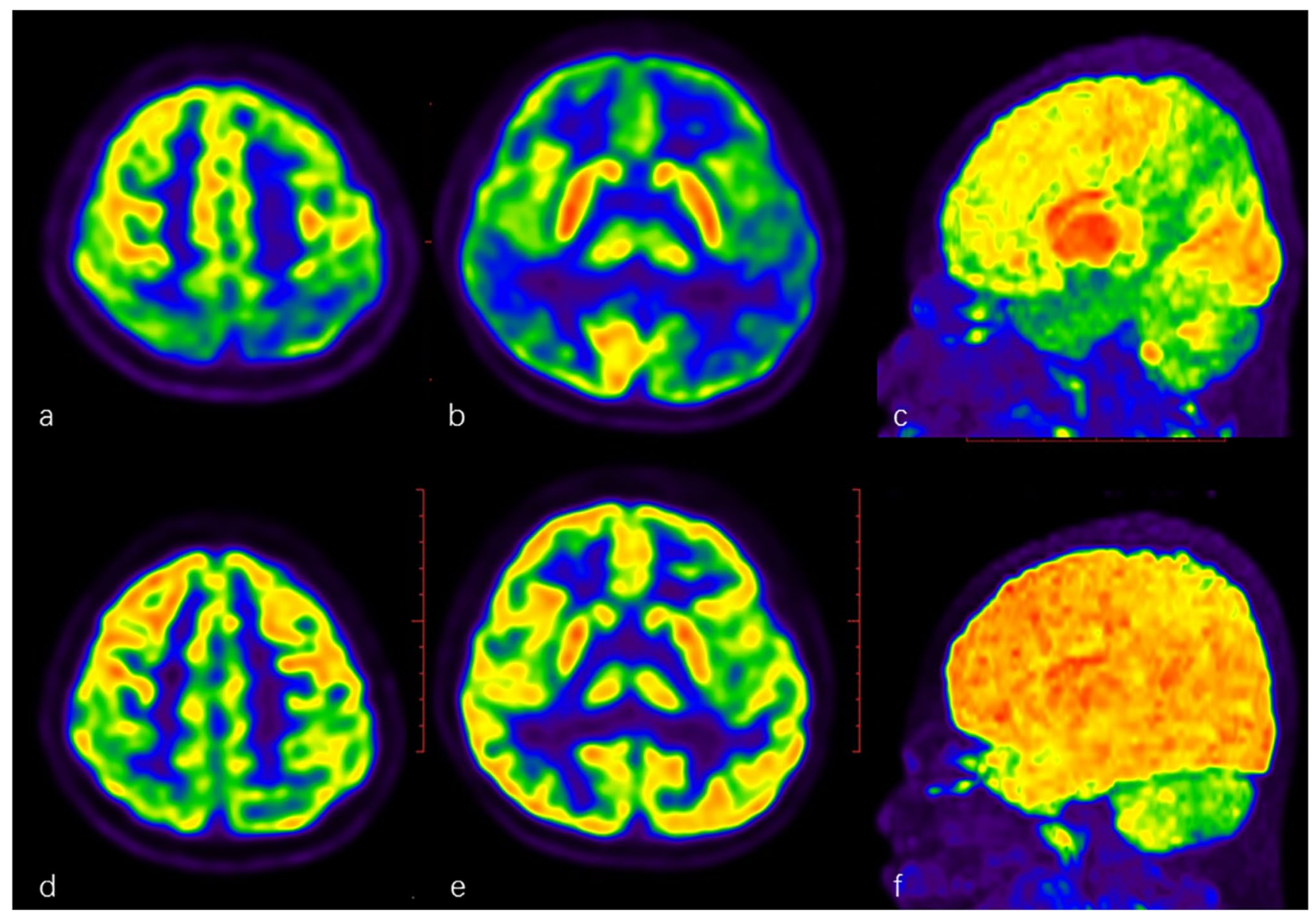

Fig. 2 A 7-year-old boy with a 6-year history of seizures and developmental retardation was finally diagnosed with seronegative AE. Brain ${ }^{18}$ F-FDG PET/CT showed large-scale hypometabolism in bilateral parietal, temporal, and frontal lobes with focal hypermetabolism in basal ganglia (a, b, c). 2.5 months later, the second brain ${ }^{18} \mathrm{~F}-\mathrm{FDG}$ PET/CT scanning indicated the significant recovery of the abnormal metabolism (d, e, f) after immunotherapy, consistent with the improvement of clinical symptoms. $\mathbf{c}, \mathbf{f}$ MIP map of FDG PET

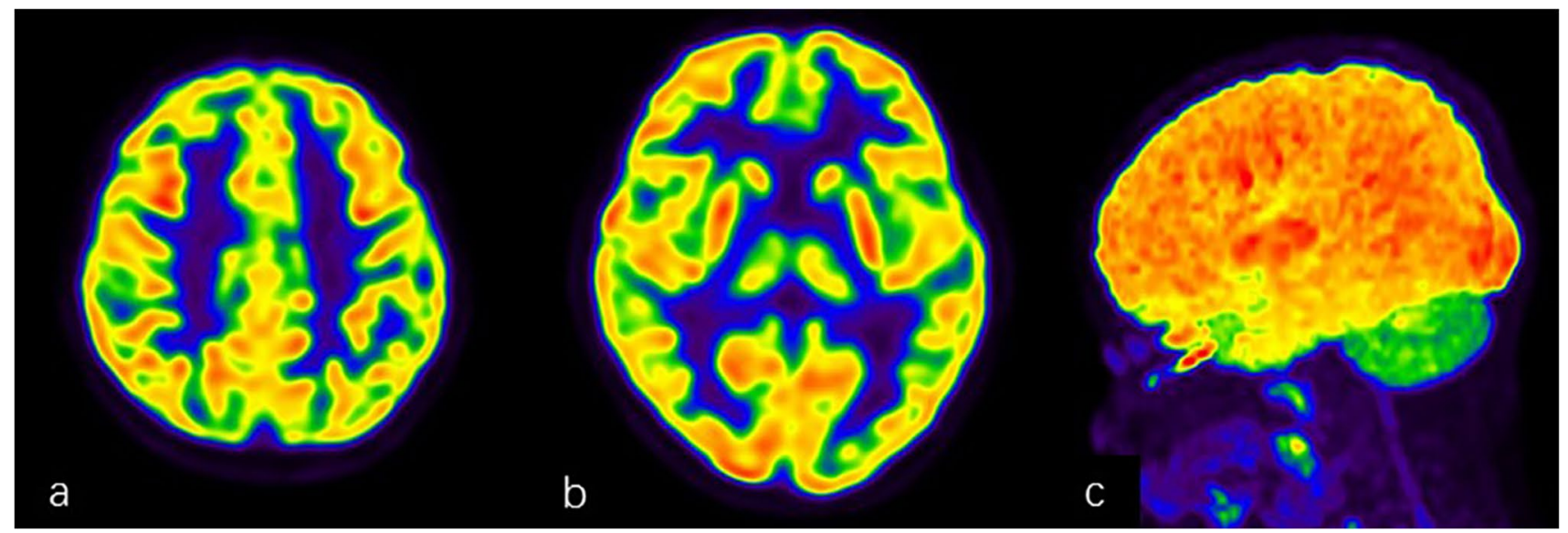

Fig. 3 A 7-year-old boy with a 5-year history of seizures presented with normal brain ${ }^{18}$ F-FDG PET/CT imaging. a, b Cross-sectional image of FDG PET. c:MIP map of FDG PET 


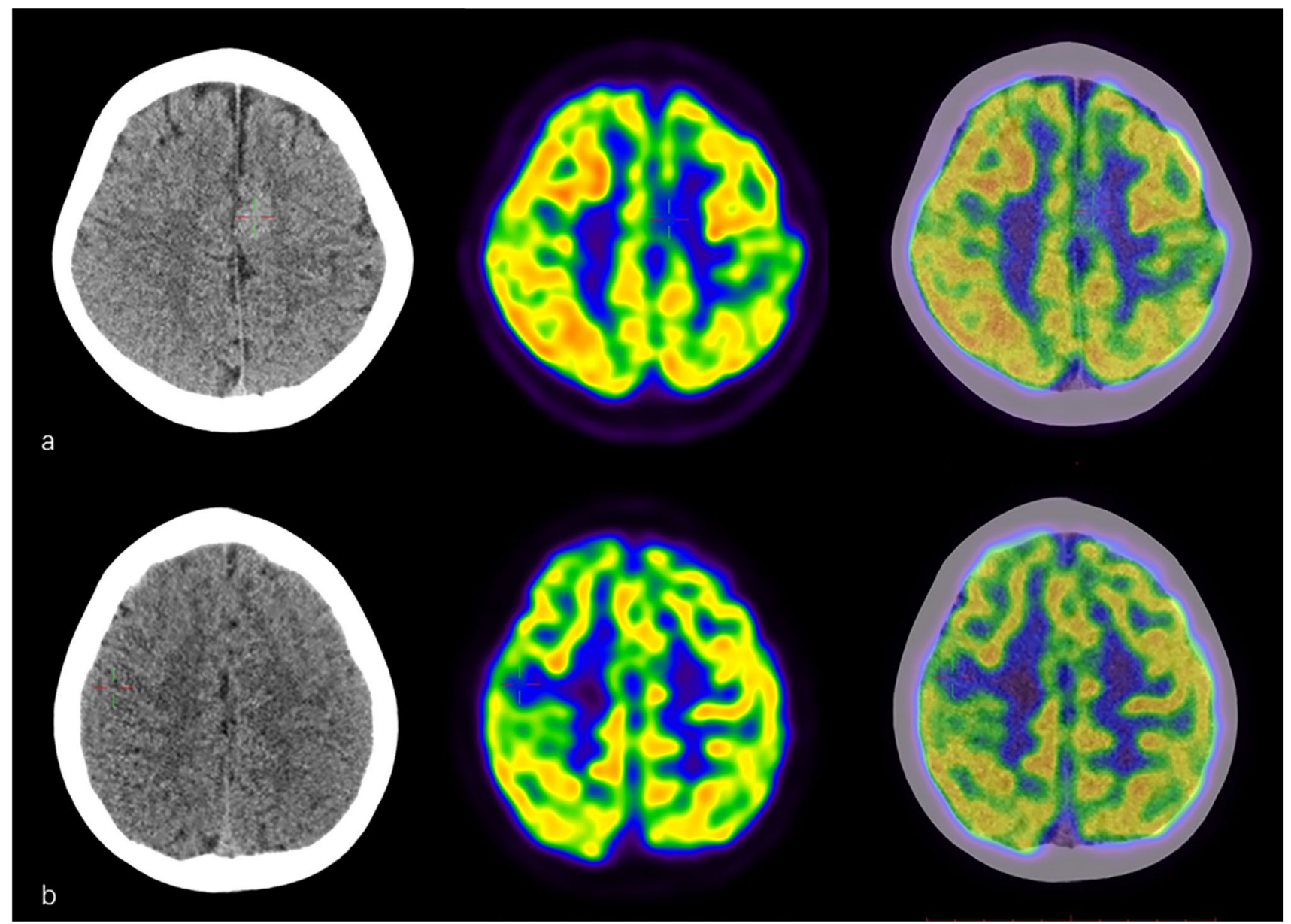

Fig. 4 a A 6-year-old boy with a 9-month history of seizures. Brain ${ }^{18}$ F-FDG PET/CT showed focal hypometabolism in left frontal lobe (the cross) with high density on CT correspondingly (the cross). The boy was finally diagnosed with frontal epilepsy. b A 6-year-old girl with a 4-month history of seizures. Brain ${ }^{18}$ F-FDG PET/CT indicated focal hypometabolism in right central sulcus mainly in the parietal lobe (the cross) with no abnormality found on CT correspondingly (the cross). The girl got well recovery after immunotherapy and was finally diagnosed with seronegative $\mathrm{AE}$

definite antibody-positive $\mathrm{AE}$ were all diagnosed with $\mathrm{AE}$ by brain FDG PET/CT imaging. Twelve of 13 children who fulfilled the criteria for possible $\mathrm{AE}$ were diagnosed with AE by FDG PET/CT imaging, and 28 of 31 children who did not meet the proposed criteria were diagnosed with AE by FDG PET/CT (Table 4).
Four children who met the criteria for probable antibodynegative $\mathrm{AE}$ and 10 children who met the criteria for

\section{Brain FDG PET/CT imaging improved the diagnosis of $A E$ by newly published diagnostic criteria for pediatric $A E$}

Table 4 FDG PET/CT

improved diagnosis of $\mathrm{AE}$ based on proposed classification criteria by AE International Working Group

\begin{tabular}{lllll}
\hline Final diagnosis & \multicolumn{4}{l}{ Classification diagnosis by AE International Working Group } \\
\cline { 2 - 5 } & Possible AE & $\begin{array}{l}\text { Probable antibody- } \\
\text { negative AE }\end{array}$ & $\begin{array}{l}\text { Definite antibody- } \\
\text { positive AE }\end{array}$ & Not met \\
\hline Seropositive AE (16) & - & - & 10 & 6 \\
Seronegative AE (42) & 13 & 4 & - & 25 \\
FDG PET/CT* (54) & $12(92.3 \%)$ & $4(100 \%)$ & $10(100 \%)$ & $28(90.3 \%)$ \\
\hline
\end{tabular}

*Abnormal metabolism on FDG PET/CT was diagnosed with AE 


\section{Discussion}

For AE, as a group of treatable acquired central nervous system disorders mostly affecting children and young people, the immunosuppression therapy, especially the early initiation of treatment, is crucial for optimal clinical outcome. In recent decade, much advances have emerged in this field, but a great part of children with suspected AE was still misdiagnosed in the initiation and missed the best time starting treatment, mostly due to having unidentified autoantibodies [2], who might be seronegative or delayed positive autoantibody test, and not meet the existing criteria, which depends on the abnormal findings of serum or CSF sampling, EEG and MRI $[12,26]$. Most of the suspected $\mathrm{AE}$ children were with various unspecific symptoms, and as has been widely reported, a large proportion of suspicions have normal or non-specific findings of serum or CSF sampling [27, 28], EEG [17], and MRI $[13,14]$.

Since the publication of a clinical approach to diagnosis of AE in the journal of Lancet Neurology in 2016, a large number of studies on FDG PET, almost all were retrospective, have been reported and indicated that FDG PET is an important biomarker for suspected AE [18]. These studies showed a much greater sensitivity for detecting abnormalities in AE than MRI [14] and other tests currently included in consensus criteria for the diagnosis of AE [17]. The predominant abnormality on brain FDG PET imaging was lobar hypometabolism, which mostly involved parietal and occipital lobes, some with hypermetabolism, mainly in basal ganglia and partial cortical lobe [14, 17, 22, 29-31].

Based on the valuable metabolic abnormalities on brain FDG PET/CT imaging from the retrospective studies in AE patients, we designed this prospective study for suspected AE children, and the diagnostic criteria for FDG $\mathrm{PET} / \mathrm{CT}$ were extensive hypometabolism in more than one lobe or one lobe with bilateral involved with or without focal hypermetabolism. The results indicated that ${ }^{18} \mathrm{~F}$-FDG $\mathrm{PET} / \mathrm{CT}$ had high specificity, sensitivity, and accuracy in diagnosing AE for suspected pediatric AE. FDG PET/ CT showed large lobar hypometabolism involved mostly in the parietal lobe, occipital and frontal lobes followed, and temporal lobe last in AE children. More than $85 \%$ of children with AE showed abnormal metabolism involved in at least two lobes. Nearly half of the children with AE showed hypermetabolic basal ganglia, and $13.8 \%$ showed hypermetabolism in focal cortex on FDG PET/CT, which was similar to the previous report $[22,29,30]$. In total, nearly up to $60 \%$ of children with AE showed lobar hypometabolism with focal hypermetabolism.

In this prospective study, the positive rate of FDG PET/ $\mathrm{CT}$ in $\mathrm{AE}$ children was $93.1 \%$ on visual assessment, which was similar to the reported data $(85-100 \%)[14,17,30]$. In fact, our positive rate was only about lobar abnormalities excluding focal hypometabolism; in this point, it was different from those reported retrospective studies. Previous studies have shown that the autoimmune antibodies can directly disrupt the synaptic function of affected neurons $[32,33]$, which can explain the lobar hypometabolism in AE patients. Anyway, hypometabolism can occur in many diseases, such as stroke, tumor, and epilepsy. Stroke and tumor usually present with regional decreased glucose metabolism and unilateral foci and could be easily identified by CT or MR. The patients with epilepsy mostly present with unilateral focal hypometabolism in interictal stage and focal hypermetabolism in ictal stage, although some cases were accompanied with extensive area of hypometabolism in addition to bilateral thalamic and cerebellar hypometabolic changes [34, 35]. Large lobar hypometabolism, more than one lobe, at least bilateral lobes, seemed more commonly observed in AE patients, which could be considered the specific findings on FDG PET/ CT for patients with suspected AE. Of course, hypermetabolism in basal ganglia and/or cortex is also a specific finding accompanied with lobar hypometabolism, which happened in more than half cases.

CSF sampling, EEG, and MRI were also analyzed in this study. Although there was significant difference between $\mathrm{AE}$ and non-AE, the positive rate of CSF abnormalities was only $58.6 \%$ in AE children, much lower than FDG PET/CT with $93.1 \%$. As reported, $56 \%$ of patients with AE could have CSF pleocytosis, but about $30 \%$ of patients with AE had a "normal" CSF, which is considered the key element of proposed diagnostic criteria for possible AE [27, 28]. The positive rate of EEG was over $60 \%$ in both $\mathrm{AE}$ and non-AE groups.

In our study, the positive rate of MRI was lower than some reports, and no specific AE abnormalities were found except for one girl who showed specific abnormal changes on MRI, but the girl was diagnosed with brain tumor in the end. Usually, if the children presented specific abnormalities of AE on MRI and got the right diagnosis and treatment, few would visit our hospital. This could, at least in part, explain the low finding rate of specific abnormalities on MRI in this study. Nevertheless, all the reported data indicated that the positive rate of specific findings on MRI was very low in AE patients [14-16], that is to say that most of the hypometabolism involved regions did not show abnormalities on MRI, suggesting the dysfunction of neurons was in the absence of structural disorders. As reported in this study, extensive hypometabolism in multiple lobes likely reflects widespread impairment of neuronal activity [36], exactly synaptic activity in AE, based on glucose consumption at synapses increasing in proportion of neuronal activity [34] and the evidence of 
autoimmune antibodies directly disrupting the synaptic function of affected neurons $[32,33]$.

Seizures were found in $59.2 \%$ recruited children with suspected $\mathrm{AE}$, and $55.2 \%$ of $\mathrm{AE}$ children presented with seizures in this study. As reported, seizures were thought to be the predominant manifestation in $\mathrm{AE}$ [26]. In a very recent meta-analysis, the overall incidence of AE patients with seizures was $42 \%$, and in the anti-NMDAR encephalitis patients, the rate increased to 73\% [37]. Anti-NMDAR encephalitis is the most common type of $\mathrm{AE}$ in pediatric patients. Among the 104 recruited children, 23 children, nearly half of non-AE patients, were eventually diagnosed with epilepsy. FDG PET/CT found the epileptogenic foci in 10 children successfully. In this study, neuropsychiatric changes were found at a higher rate of $70.7 \%$ than seizures in AE children. The children presenting with both seizures and neuropsychiatric changes were more likely diagnosed with AE compared with non-AE.

In this study, 9 children underwent the repeated FDG PET/CT scanning after immunotherapy, two-thirds of them demonstrated good recovery on PET imaging 1 to 7 months after therapy, showing excellent evidence of the recovery of neuronal synaptic function. One-third showed more obvious abnormal metabolism on FDG PET imaging after a short time, which might be due to the different clinical course. Some studies showed the variable brain metabolic patterns might be associated with the different clinical course; the cortical hypometabolism might be more extensive in early recovery phase [29].

In our study, 31 pediatric patients with AE did not meet the latest diagnostic criteria of $\mathrm{AE}$ in the pediatric patient [26], while $90.3 \%$ of them were correctly diagnosed by brain FDG PET/CT imaging. Similarly, $92.3 \%$ of patients who fulfilled the criteria for possible pediatric $\mathrm{AE}$ were correctly diagnosed with AE by brain FDG PET/CT imaging. The most common reasons that more than half of pediatric patients with $\mathrm{AE}$ did not meet the proposed diagnostic criteria were long onset of symptoms, normal or non-specific MRI and CSF findings, and the absence of brain biopsy. Similar reports about the application of proposed diagnostic criteria were published in adult AE. Giordano et al. [38] reported that $30.3 \%$ of included adult AE patients did not meet the criteria for definite or probable AE. Another report showed nearly $15 \%$ patients did not meet the criteria for possible AE, and of the patients with anti-NMDAR encephalitis, $17 \%$ did not fulfil the diagnostic criteria for possible AE, and $25 \%$ failed to meet the criteria for probable anti-NMDAR encephalitis [39]. Based on our study, it is suggested that when pediatric patients with suspected AE failed to meet the criteria for probable or definite $\mathrm{AE}$, especially with normal or non-specific CSF and MRI findings and the absence of a brain biopsy, brain FDG PET/CT imaging is strongly recommended.
Some limitations in this study need to be mentioned. First, the recruited children were all at least one-year-old. There was no data analyzed about infants younger than oneyear-old due to infant brain being in development with variable glucose metabolism in the cortex. Second, there was no quantitative statistical software adopted due to no normal children's brain FDG PET imaging template. Although some retrospective studies reported using the adult brain FDG PET template, it seemed not precise and critical meaningful. The normal template of children is really hard or almost impossible to obtain for ethical consideration.

\section{Conclusion}

This prospective study indicated that brain FDG PET/CT was very useful for clinically suspected AE children, with high specificity, sensitivity, and accuracy for diagnosis of AE. The typical findings, large lobar hypometabolism, mostly accompanied with hypermetabolism in basal ganglia and/or focal cortex, on FDG PET/CT were strong supporting criteria for $\mathrm{AE}$ diagnosis in suspected children. Overall, brain FDG PET/CT should be considered to be included in the diagnostic criteria for $\mathrm{AE}$ in children, especially for those with negative neuronal autoantibodies, and normal CSF and non-specific brain MRI findings, who failed to meet the criteria for probable AE.

Author contribution Dr. Yin had full access to all the data in the study and takes responsibility for the integrity of the data and the accuracy of the data analysis. Concept and design: Yafu Yin, Hui Wang, Ling Li. Acquisition, analysis, or interpretation of data: Yafu Yin, Jing Wu, Shuqi Wu, Suyun Chen, Weiwei Cheng. Drafting of the manuscript: Yafu Yin, Jing Wu. Critical revision of the manuscript for important intellectual content: Yafu Yin, Weiwei Cheng. Statistical analysis: Yafu Yin, Suyun Chen, Shuqi Wu. All authors read and approved the final manuscript.

Funding This work was supported by the National Nature Science Foundation of China (No. 81974270) and Shanghai Pujiang Program (2019PJD032).

Data availability The datasets generated during and/or analyzed during the current study are available from the corresponding author on reasonable request.

\section{Declarations}

Ethics approval The study was approved by the Ethics Committee of Xinhua Hospital Affiliated to Shanghai Jiao Tong University School of Medicine.

Consent to participate Written informed consent was obtained from the parents.

Consent for publication Not applicable. 
Competing interests The authors declare no competing interests.

Open Access This article is licensed under a Creative Commons Attribution 4.0 International License, which permits use, sharing, adaptation, distribution and reproduction in any medium or format, as long as you give appropriate credit to the original author(s) and the source, provide a link to the Creative Commons licence, and indicate if changes were made. The images or other third party material in this article are included in the article's Creative Commons licence, unless indicated otherwise in a credit line to the material. If material is not included in the article's Creative Commons licence and your intended use is not permitted by statutory regulation or exceeds the permitted use, you will need to obtain permission directly from the copyright holder. To view a copy of this licence, visit http://creativecommons.org/licenses/by/4.0/.

\section{References}

1. Dalmau J, Graus F. Antibody-Mediated Encephalitis. N Engl J Med. 2018;378:840-51.

2. Dale RC, Gorman MP, Lim M. Autoimmune encephalitis in children: clinical phenomenology, therapeutics, and emerging challenges. Curr Opin Neurol. 2017;30:334-44.

3. Qiu X, Zhang H, Li D, Wang J, Jiang Z, Zhou Y, et al. Analysis of clinical characteristics and poor prognostic predictors in patients with an initial diagnosis of autoimmune encephalitis. Front Immunol. 2019;10:1286.

4. Balu R, McCracken L, Lancaster E, Graus F, Dalmau J, Titulaer MJ. A score that predicts 1-year functional status in patients with anti-NMDA receptor encephalitis. Neurology. 2019;92:e244-52.

5. Finke C, Pruss H, Heine J, Reuter S, Kopp UA, Wegner F, et al. Evaluation of cognitive deficits and structural hippocampal damage in encephalitis with leucine-rich, glioma-inactivated 1 antibodies. JAMA Neurol. 2017;74:50-9.

6. Hebert J, Day GS, Steriade C, Wennberg RA, Tang-Wai DF. Longterm cognitive outcomes in patients with autoimmune encephalitis. Can J Neurol Sci. 2018;45:540-4.

7. Irani SR, Stagg CJ, Schott JM, Rosenthal CR, Schneider SA, Pettingill $\mathrm{P}$, et al. Faciobrachial dystonic seizures: the influence of immunotherapy on seizure control and prevention of cognitive impairment in a broadening phenotype. Brain. 2013;136:3151-62.

8. Irani SR, Gelfand JM, Al-Diwani A, Vincent A. Cell-surface central nervous system autoantibodies: clinical relevance and emerging paradigms. Ann Neurol. 2014;76:168-84.

9. Vollmer TL, McCarthy M. Autoimmune encephalitis: a more treatable tragedy if diagnosed early. Neurology. 2016;86:1655-6.

10. Lee WJ, Lee ST, Byun JI, Sunwoo JS, Kim TJ, Lim JA, et al. Rituximab treatment for autoimmune limbic encephalitis in an institutional cohort. Neurology. 2016;86:1683-91.

11. Titulaer MJ, McCracken L, Gabilondo I, Armangue T, Glaser C, Iizuka $\mathrm{T}$, et al. Treatment and prognostic factors for long-term outcome in patients with anti-NMDA receptor encephalitis: an observational cohort study. Lancet Neurol. 2013;12:157-65.

12. Graus F, Titulaer MJ, Balu R, Benseler S, Bien CG, Cellucci T, et al. A clinical approach to diagnosis of autoimmune encephalitis. Lancet Neurol. 2016;15:391-404.

13. Baumgartner A, Rauer S, Mader I, Meyer PT. Cerebral FDG-PET and MRI findings in autoimmune limbic encephalitis: correlation with autoantibody types. J Neurol. 2013;260:2744-53.

14. Solnes LB, Jones KM, Rowe SP, Pattanayak P, Nalluri A, Venkatesan A, et al. Diagnostic value of (18)F-FDG PET/CT versus MRI in the setting of antibody-specific autoimmune encephalitis. J Nucl Med. 2017;58:1307-13.
15. Newey CR, Sarwal A, Hantus S. [(18)F]-Fluoro-deoxy-glucose positron emission tomography scan should be obtained early in cases of autoimmune encephalitis. Autoimmune Dis. 2016;2016:9450452.

16. Ances BM, Vitaliani R, Taylor RA, Liebeskind DS, Voloschin A, Houghton DJ, et al. Treatment-responsive limbic encephalitis identified by neuropil antibodies: MRI and PET correlates. Brain. 2005; 128:1764-77.

17. Probasco JC, Solnes L, Nalluri A, Cohen J, Jones KM, Zan E, et al. Abnormal brain metabolism on FDG-PET/CT is a common early finding in autoimmune encephalitis. Neurol Neuroimmunol Neuroinflamm. 2017;4:e352.

18. Morbelli S, Djekidel M, Hesse S, Pagani M, Barthel H, Neuroimaging Committee of the European Association of Nuclear M, et al. Role of (18)F-FDG-PET imaging in the diagnosis of autoimmune encephalitis. Lancet Neurol. 2016;15:1009-10.

19. Sekigawa M, Okumura A, Niijima S, Hayashi M, Tanaka K, Shimizu T. Autoimmune focal encephalitis shows marked hypermetabolism on positron emission tomography. J Pediatr. 2010;156:158-60.

20. Kojima G, Inaba M, Bruno MK. PET-positive extralimbic presentation of anti-glutamic acid decarboxylase antibody-associated encephalitis. Epileptic Disord. 2014;16:358-61.

21. Endres D, Perlov E, Stich O, Rauer S, Maier S, Waldkircher Z, et al. Hypoglutamatergic state is associated with reduced cerebral glucose metabolism in anti-NMDA receptor encephalitis: a case report. BMC Psychiatry. 2015;15:186.

22. Novy J, Allenbach G, Bien CG, Guedj E, Prior JO, Rossetti AO. FDG-PET hyperactivity pattern in anti-NMDAr encephalitis. J Neuroimmunol. 2016;297:156-8.

23. Jang Y, Lee ST, Bae JY, Kim TJ, Jun JS, Moon J, et al. LGI1 expression and human brain asymmetry: insights from patients with LGI1-antibody encephalitis. J Neuroinflammation. 2018;15:279.

24. Moubtakir A, Dejust S, Godard F, Messaoud L, Morland D. 18F-FDG PET/CT in Anti-NMDA Receptor Encephalitis: Typical Pattern and Follow-up. Clin Nucl Med. 2018;43:520-1.

25. Morbelli S, Arbizu J, Booij J, Chen MK, Chetelat G, Cross DJ, et al. The need of standardization and of large clinical studies in an emerging indication of [(18)F]FDG PET: the autoimmune encephalitis. Eur J Nucl Med Mol Imaging. 2017;44:353-7.

26. Cellucci T, Van Mater H, Graus F, Muscal E, Gallentine W, Klein-Gitelman MS, et al. Clinical approach to the diagnosis of autoimmune encephalitis in the pediatric patient. Neurol Neuroimmunol Neuroinflamm. 2020;7.

27. Hebert J, Gros P, Lapointe S, Amtashar FS, Steriade C, Maurice $\mathrm{C}$, et al. Searching for autoimmune encephalitis: Beware of normal CSF. J Neuroimmunol. 2020;345:577285.

28. Dubey D, Pittock SJ, Kelly CR, McKeon A, Lopez-Chiriboga AS, Lennon VA, et al. Autoimmune encephalitis epidemiology and a comparison to infectious encephalitis. Ann Neurol. 2018;83:166-77.

29. Yuan J, Guan H, Zhou X, Niu N, Li F, Cui L, et al. Changing brain metabolism patterns in patients with ANMDARE: serial 18F-FDG PET/CT findings. Clin Nucl Med. 2016;41:366-70.

30. Turpin S, Martineau P, Levasseur MA, Meijer I, Decarie JC, Barsalou J, et al. 18F-Flurodeoxyglucose positron emission tomography with computed tomography (FDG PET/CT) findings in children with encephalitis and comparison to conventional imaging. Eur J Nucl Med Mol Imaging. 2019;46:1309-24.

31. Tripathi M, Tripathi M, Roy SG, Parida GK, Ihtisham K, Dash $\mathrm{D}$, et al. Metabolic topography of autoimmune non-paraneoplastic encephalitis. Neuroradiology. 2018;60:189-98.

32. Mikasova L, De Rossi P, Bouchet D, Georges F, Rogemond V, Didelot A, et al. Disrupted surface cross-talk between NMDA 
and Ephrin-B2 receptors in anti-NMDA encephalitis. Brain. 2012;135:1606-21.

33. Hughes EG, Peng X, Gleichman AJ, Lai M, Zhou L, Tsou R, et al. Cellular and synaptic mechanisms of anti-NMDA receptor encephalitis. J Neurosci. 2010;30:5866-75.

34. Wahl RL. Principles and Practice of PET and PET/CT. 2nd ed: Lippincott Williams \& Wilkins; 2009.

35. Tian M, Watanabe Y, Kang KW, Murakami K, Chiti A, Carrio I, et al. International consensus on the use of [(18)F]-FDG PET/ $\mathrm{CT}$ in pediatric patients affected by epilepsy. Eur J Nucl Med Mol Imaging. 2021.

36. Dalmau J, Lancaster E, Martinez-Hernandez E, Rosenfeld MR, Balice-Gordon R. Clinical experience and laboratory investigations in patients with anti-NMDAR encephalitis. Lancet Neurol. 2011;10:63-74
37. Chen L, Zhu L, Lu D, Dai S, Han Y, Wu Z, et al. Association between autoimmune encephalitis and epilepsy: Systematic review and meta-analysis. Seizure. 2021;91:346-59.

38. Giordano A, Fazio R, Gelibter S, Minicucci F, Vabanesi M, Anzalone $\mathrm{N}$, et al. Diagnosing autoimmune encephalitis in a real-world single-centre setting. J Neurol. 2020;267:449-60.

39. Baumgartner A, Rauer S, Hottenrott T, Leypoldt F, Ufer F, Hegen $\mathrm{H}$, et al. Admission diagnoses of patients later diagnosed with autoimmune encephalitis. J Neurol. 2019;266:124-32.

Publisher's note Springer Nature remains neutral with regard to jurisdictional claims in published maps and institutional affiliations. 